\title{
New roles revealed for $T$ cells and DCs in glomerulonephritis
}

\author{
Alfred H.J. Kim, ${ }^{1}$ Mary A. Markiewicz, ${ }^{2}$ and Andrey S. Shaw ${ }^{2,3}$ \\ ${ }^{1}$ Division of Rheumatology, Department of Internal Medicine, ²Division of Immunobiology, Department of Pathology and Immunology, and \\ ${ }^{3}$ Howard Hughes Medical Institute, Washington University School of Medicine, St. Louis, Missouri, USA.
}

\begin{abstract}
Little is known about the potential role of $T$ cells in the inflammatory renal disease glomerulonephritis (GN). GN has been historically viewed as a product of immune complex-mediated complement activation, and the presence of autoantibodies made identifying $T$ cell-specific effector contributions difficult to elucidate. In this issue of the JCI, Heymann et al. generate what they believe to be a novel, transgenic murine model of GN, demonstrating a direct role for $\mathrm{CD8}^{+} \mathrm{T}$ cells, activated $\mathrm{CD} 4^{+} \mathrm{T}$ cells, and DCs in the pathogenesis of GN (see the related article beginning on page 1286).
\end{abstract}

The glomerulonephritides represent a diverse pathological and clinical subset of kidney diseases sharing the common endpoint of glomerular inflammation. It has been long assumed that deposition of immune complexes and subsequent complement activation were requisite steps in initiating disease. Recent data using mouse models, however, suggest that the pathogenesis of glomerular injury is more complicated. Mice deficient in various complement proteins still generate inflammation in a classic model of immune complex-triggered inflammation, the Arthus reaction (1-3). The absence of certain Fc receptors, such as Fc $\gamma$ RIII, abrogates glomerulonephritis $(\mathrm{GN})$, even in the presence of immune complex deposition and complement activation in glomeruli (4), via loss of neutrophil (5) and macrophage (6) effector functions.

Over the last ten years, evidence has been slowly accumulating that suggests a potential role for $\mathrm{T}$ cells in GN (7-9). Nevertheless, the idea of a causal role for $\mathrm{T}$ cells in GN remains weakly accepted, since much critical information is lacking. For example, we still do not know what role these cells play in the induction of GN or what the potential autoantigens are. In this issue of the JCI, Heymann and colleagues take our understanding of GN a considerable step forward by developing

Conflict of interest: A.S. Shaw is a consultant for Pfizer Inc.

Nonstandard abbreviations used: GBM, glomerular basement membrane; GN, glomerulonephritis.

Citation for this article: J. Clin. Invest. 119:1074-1076 (2009). doi:10.1172/JCI39071. a model of experimental GN based on two model antigens (10). The authors generated transgenic mice expressing the model antigens OVA and hen egg lysozyme in kidney podocytes. This allowed for the use of the well-characterized OVA-transgenic T cells: OVA-specific $\mathrm{CD}^{+} \mathrm{T}$ cells (OT-I cells) and OVA-specific CD4 ${ }^{+} \mathrm{T}$ helper cells (OT-II cells). The model allowed them to establish a causal role for $T$ cells in mediating glomerular destruction.

\section{Direct role of T cells in glomerular immunopathology}

Prior to this study (10), experimental autoimmune GN (EAG) represented the most relevant model for examining the contribution of T cells to GN. In this model, mice immunized with purified glomerular basement membranes (GBMs) developed GN. With the identification of the Goodpasture antigen (11) - the noncollagenous domain of the $\alpha 3$ chain of type 4 collagen $(\alpha 3$ IV-NC1) - a specific antigen could also be used to induce disease. While initial studies demonstrated that transfer of serum from immunized animals could recapitulate disease, depletion of $\mathrm{CD}^{+} \mathrm{T}$ cells attenuated GN pathology and autoantibody production (12). These studies were interpreted to support a primary role for autoantibodies, with $\mathrm{T}$ cells functioning mainly to provide B cell help $(7,8)$. Subsequent work suggested that $\mathrm{T}$ cells played a requisite role in pathogenesis, as serum could not initiate disease in $\mathrm{T}$ cell receptor-deficient animals (9). Furthermore, adoptive transfer of GBMspecific $T$ cells generated in immunized mice conferred GN in recipient mice (9).
Interference with the function (13), activation (14), or adherence (15) of T cells was also shown to attenuate EAG activity. The exact role of $\mathrm{T}$ cells in glomerular immunopathology is unclear, but two possible mechanisms include macrophage recruitment to the inflamed kidney mediated by Th1 cells (16) and epitope spreading caused by $\mathrm{T}$ cell-mediated damage and antigen release (17).

To begin analyzing the specific roles of T cells, Heymann et al. (10) first injected naive, OVA-specific CD8 ${ }^{+} \mathrm{T}$ cells into their transgenic mice. These cells proliferated in kidney draining lymph nodes, suggesting that OVA expressed on podocytes is taken up by DCs that migrate to the draining renal lymph nodes. These activated CTLs, however, did not cause disease, presumably because they were unable to traffic back into the noninflamed kidney. The authors then tested whether the addition of $\mathrm{CD}^{+} \mathrm{T}$ cells to the $\mathrm{CD}^{+} \mathrm{T}$ cells would allow for the development of GN. While the addition of naive $\mathrm{CD}^{+} \mathrm{T}$ cells had no effect, the addition of activated OVAspecific $\mathrm{CD}^{+} \mathrm{T}$ cells with the naive $\mathrm{CD}^{+}$ $\mathrm{T}$ cells resulted in accumulation of both $\mathrm{CD}^{+}$and $\mathrm{CD}^{+} \mathrm{T}$ cells in the kidney and development of GN.

Why the addition of ex vivo-activated $\mathrm{CD}^{+} \mathrm{T}$ cells was required in the current study is unclear (10), but a similar requirement has been reported in a model of diabetes in which OVA is expressed in pancreatic $\beta$ cells (18-20). This could be related to the findings of Heymann et al. (10) that naive, OVA-specific CD4 ${ }^{+} \mathrm{T}$ cells did not proliferate when transferred into the transgenic mice, explaining the need to activate these cells ex vivo. The inability to activate $\mathrm{CD}^{+} \mathrm{T}$ cells in vivo could be related to low antigen levels or to the type of DC carrying the podocyte antigens to the lymph node. Hopefully, this issue will be explored in future experiments. It will also be important to test the general conclusions of their study using another model antigen system. 


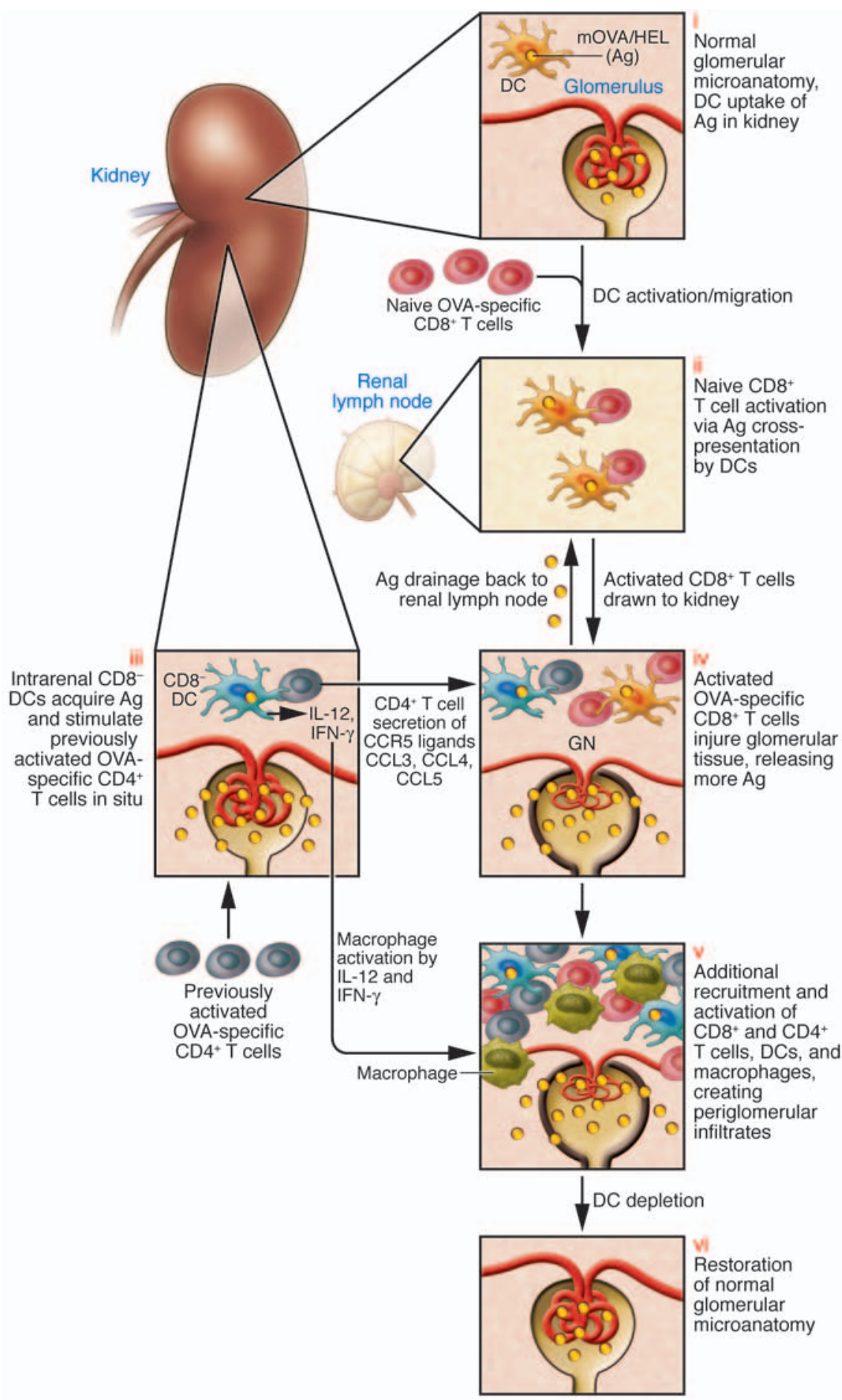

Nonetheless, the data reported by Heymann et al. (10) clearly demonstrate that the infiltration of activated $\mathrm{CD} 4^{+} \mathrm{T}$ cells into the kidney is required for the trafficking of CTLs to the kidney. One mechanism for the recruitment of CTLs explored by the authors is the production of chemokines capable of recruiting CTLs (CCL3, CCL4, and CCL5). Indeed, they found that antigen-specific $\mathrm{CD}^{+} \mathrm{T}$ cells purified from the kidney produced greater amounts of Ccl3, Ccl4, and Ccl5 mRNA. As the $\mathrm{CD}^{+}$ $\mathrm{T}$ cells in the kidney were also producing Th1 cytokines, the authors imply that this subset may be important to confer renal pathology. Nevertheless, it will be important to directly test this using other Th subsets, such as Th2 or Th17, and Tregs.

\section{Figure 1}

The potential role of T cells and DCs in GN. In the report by Heymann et al. in this issue of the $\mathrm{JCl}(10)$, a membrane-tethered form of OVA/hen egg lysozyme (mOVA/HEL) fusion antigen $(\mathrm{Ag})$ expressed by glomerular podocytes, generated T cell- and DC-mediated GN in mice. (i) Antigen produced by podocytes is taken up by DCs in the kidney. (ii) Upon transfer of naive, OVA-specific CD ${ }^{+}$ $T$ cells to these transgenic mice, DCs within renal lymph nodes activate these $T$ cells, causing clonal proliferation via antigen crosspresentation. (iii) Previously activated OVAspecific $C D 4^{+} T$ cells become further activated within the renal tissue by resident CD8- DCs, which induces CCR5 ligand expression by CD4 ${ }^{+} \mathrm{T}$ cells and IL-12 secretion by DCs. The presence of CCR5 ligands draws activated $\mathrm{CD}^{+} \mathrm{T}$ cells from the renal lymph node to the kidney, and the IL-12 further promotes CTL effector activity. (iv) Lysis of OVA-bearing cells by $\mathrm{CD}^{+} \mathrm{T}$ cells releases more antigen, which is endocytosed by resident CD8- DCs or drains to the renal lymph nodes, augmenting the response. (v) Additional macrophages, CD4 ${ }^{+} \mathrm{T}$ cells, CD8 ${ }^{+} \mathrm{T}$ cells, and $\mathrm{DCs}$ are recruited into the periglomerular space, further inducing glomerular injury. (vi) Depletion of diphtheria toxin-sensitive DCs after establishment of GN completely reverses immune infiltration pathology and restores normal glomerular microanatomy.

The beauty of the model is that it possesses tremendous flexibility in allowing the contribution of various Th cells in GN to be determined. In vitro differentiated Th subsets can be administered, yielding important conclusions regarding the role of Th subsets in autoimmune pathology.

\section{DCs play a central role in mediating periglomerular infiltrates}

DCs are a functionally diverse class of antigen-presenting cells found in many tissues that possess tremendous immunostimulatory potential due to their ability to active $\mathrm{T}$ cells. DCs are constitutively present in the glomeruli (21), but their role in GN prior to the development of the model reported in this issue by Heymann et al. (10) was unknown. A striking observation made in this study was the absolute requirement for DCs within the kidney for CTL-mediated disease. Depletion of DCs from the mouse resulted in the complete disappearance of $\mathrm{T}$ cells in the kidney within 40 hours. The authors suggest that antigen-bearing DCs in the kidney are required to maintain the presence of activated $\mathrm{CD}^{+} \mathrm{T}$ cells, which in turn recruit CTLs to the kidney. Since the 
addition of activated $\mathrm{CD} 4^{+} \mathrm{T}$ cells resulted in upregulation of CD40, CD86, and IL-12 on the kidney DCs, the authors additionally suggest that the activated $\mathrm{CD} 4^{+} \mathrm{T}$ cells can induce DC maturation.

What mediates the recruitment of activated $\mathrm{CD}^{+} \mathrm{T}$ cells to the kidney? The authors suggested that activated $\mathrm{CD} 4^{+} \mathrm{T}$ cells home to kidneys but do not stay due to the absence of CTL-mediated antigen release from the glomeruli. Once CTLs induce glomerular damage, kidney DCs present antigen to $\mathrm{CD} 4^{+} \mathrm{T}$ cells and retain them in the kidney. More importantly, it will be valuable to determine what kind of DCs are involved and exactly what their role is. While the glomerular DCs might seem to be the most likely DC type involved in the processing of podocyte antigens, the periglomerular location of the inflammation suggests that the involvement of DCs is more complicated. The authors suggest that since there is a large increase in monocyte-derived inflammatory DCs (CD $11 \mathrm{c}^{+} / \mathrm{CD} 11 \mathrm{~b}^{\mathrm{hi}}$ / $\left.\mathrm{Gr}^{+}\right)$in the inflamed kidney, inflammatory DCs and not resident DCs are the likely culprit. But much more work, including kinetic studies, is necessary to demonstrate this point.

From their data (10), the Heymann et al. deduce the following testable model (Figure 1). First, the autoantigen, a membranetethered form of OVA (mOVA) expressed on podocytes, is taken up by DCs that then traffic to draining renal lymph nodes, in which they can activate naive $\mathrm{CD}^{+} \mathrm{T}$ cells. Second, activated $\mathrm{CD}^{+} \mathrm{T}$ cells home to the kidney, presumably via the recognition of OVA on antigen-presenting cells in the kidney. Third, recognition of antigen induces the expression of chemokines (CCL3, CCL4, and CCL5) that function to recruit CTLs to the kidney. Fourth, activated CTLs generate tissue damage, causing more antigen release. Fifth, released antigen is taken up by DCs that present antigen to $\mathrm{CD}^{+} \mathrm{T}$ cells in the kidney and traffic to the draining renal lymph nodes to activate more $\mathrm{CD} 8^{+} \mathrm{T}$ cells, thus amplifying the response. Finally, the intrarenal CD $4^{+}$Th 1 cells can also attract macrophages into the glomeruli to further mediate injury and fibrosis.

It is important to note also that the nephrin promoter is active in lymph nodes, including in DCs (22), which could render the podocyte-specific expression of antigen irrelevant. However, OT-I cells do not proliferate in nondraining lymph nodes, mak- ing it unlikely that antigen resides constitutively in the lymph nodes of these mice.

\section{A renewed interest in examining T cell-mediated damage in GN}

The data generated from this model (10) demonstrate that $\mathrm{T}$ cells and DCs can mediate GN in the absence of immune complex-mediated pathology. DCs control the presence of $\mathrm{T}$ cell infiltration, while $\mathrm{CD}^{+} \mathrm{T}$ cells potentiate the severity of cellular infiltration and $\mathrm{CD}^{+} \mathrm{T}$ cells confer glomerular injury. Activated macrophages also infiltrate the kidney in a T cell-dependent manner, providing additional effector mechanisms for glomerular injury.

Although much can be learned from this model, podocyte-derived antigen expression may not represent a physiologically relevant system for examining GN. Virtually all putative antigens thought to play a role in GN trap themselves against the GBM, as with nucleic acids, or are part of the GBM itself. It remains to be determined whether GN induced by podocyte antigens shows similar immunopathologies to that induced by GBM-based antigens. It will also be important to determine how autoantibodies and immune complexes may contribute to the cellular mechanisms studied here.

As what is believed to be the first model to test the role of $\mathrm{T}$ cells in the context of a model antigen, the report by Heymann et al. (10) continues to modify our understanding of $\mathrm{T}$ cell immunity in GN. This model represents the clearest example of the causative roles $\mathrm{T}$ cells play in GN pathology. Additionally, the data suggest that therapies aimed at DCs may be a promising approach for the control of acute and chronic inflammation. The design of new therapies for treating glomerular injury will need to account for cell-mediated contributions, thanks to clever models such as this.

Address correspondence to: Andrey S. Shaw, Department of Pathology and Immunology, Washington University School of Medicine, Campus Box 8118, 660 South Euclid Avenue, St. Louis, Missouri 63110, USA. Phone: (314) 362-4614; Fax: (314) 362-9108; E-mail: shaw@pathology.wustl.edu.

1. Sylvestre, D., et al. 1996. Immunoglobulin G-mediated inflammatory responses develop normally in complement-deficient mice. J. Exp. Med. 184:2385-2392.

2. Sylvestre, D.L., and Ravetch, J.V. 1994. Fc receptors initiate the Arthus reaction: redefining the inflammatory cascade. Science. 265:1095-1098.

3. Clynes, R., and Ravetch, J.V. 1995. Cytotoxic antibodies trigger inflammation through $\mathrm{Fc}$ receptors.
Immunity. 3:21-26.

4. Clynes, R., Dumitru, C., and Ravetch, J.V. 1998. Uncoupling of immune complex formation and kidney damage in autoimmune glomerulonephritis. Science. 279:1052-1054.

5. Coxon, A., et al. 2001. Fc gamma RIII mediates neutrophil recruitment to immune complexes. A mechanism for neutrophil accumulation in immunemediated inflammation. Immunity. 14:693-704.

6. Kaneko, Y., Nimmerjahn, F., Madaio, M.P., and Ravetch, J.V. 2006. Pathology and protection in nephrotoxic nephritis is determined by selective engagement of specific Fc receptors. J. Exp. Med. 203:789-797.

7. Bolton, W.K., Benton, F.R., and Lobo, P.I. 1978. Requirement of functional T-cells in the production of autoimmune glomerulotubular nephropathy in mice. Clin. Exp. Immunol. 33:474-477.

8. Reynolds, J., Sallie, B.A., Syrganis, C., and Pusey, C.D. 1993. The role of T-helper lymphocytes in priming for experimental autoimmune glomerulonephritis in the BN rat. J. Autoimmun. 6:571-585.

9. Kalluri, R., Danoff, T.M., Okada, H., and Neilson, E.G. 1997. Susceptibility to anti-glomerular basement membrane disease and Goodpasture syndrome is linked to MHC class II genes and the emergence of $\mathrm{T}$ cell-mediated immunity in mice. J. Clin. Invest. 100:2263-2275.

10. Heymann, F., et al. 2009. Kidney dendritic cell activation is required for progression of renal disease in a mouse model of glomerular injury. J. Clin. Invest. 119:1286-1297.

11. Wieslander, J., Bygren, P., and Heinegard, D. 1984. Isolation of the specific glomerular basement membrane antigen involved in Goodpasture syndrome. Proc. Natl. Acad. Sci. U. S. A. 81:1544-1548.

12. Reynolds, J., and Pusey, C.D. 1994. In vivo treatment with a monoclonal antibody to T helper cells in experimental autoimmune glomerulonephritis in the BN rat. Clin. Exp. Immunol. 95:122-127.

13. Reynolds, J., Cashman, S.J., Evans, D.J., and Pusey, C.D. 1991. Cyclosporin A in the prevention and treatment of experimental autoimmune glomerulonephritis in the brown Norway rat. Clin. Exp. Immunol. 85:28-32.

14. Reynolds, J., et al. 2000. CD28-B7 blockade prevents the development of experimental autoimmune glomerulonephritis. J. Clin. Invest. 105:643-651.

15. Nishikawa, K., et al. 1993. Antibodies to intercellular adhesion molecule $1 /$ lymphocyte functionassociated antigen 1 prevent crescent formation in rat autoimmune glomerulonephritis. J. Exp. Med. 177:667-677.

16. Li, S., Holdsworth, S.R., and Tipping, P.G. 1997. Antibody independent crescentic glomerulonephritis in mu chain deficient mice. Kidney Int. 51:672-678.

17. Bolton, W.K., Chen, L., Hellmark, T., Wieslander, J., and Fox, J.W. 2005. Epitope spreading and autoimmune glomerulonephritis in rats induced by a T cell epitope of Goodpasture's antigen. J. Am. Soc. Nephrol. 16:2657-2666.

18. Kurts, C., et al. 1997. CD4+ T cell help impairs $\mathrm{CD} 8+\mathrm{T}$ cell deletion induced by cross-presentation of self-antigens and favors autoimmunity. J. Exp. Med. 186:2057-2062.

19. Kurts, C., Kosaka, H., Carbone, F.R., Miller, J.F., and Heath, W.R. 1997. Class I-restricted crosspresentation of exogenous self-antigens leads to deletion of autoreactive CD8(+) T cells. J. Exp. Med. 186:239-245.

20. Behrens, G.M., et al. 2004. Helper requirements for generation of effector CTL to islet beta cell antigens. J. Immunol. 172:5420-5426.

21. Lindquist, R.L., et al. 2004. Visualizing dendritic cell networks in vivo. Nat. Immunol. 5:1243-1250.

22. Astrom, E., et al. 2006. Nephrin in human lymphoid tissues. Cell. Mol. Life Sci. 63:498-504. 